Fachbereich Mathematik

Nr. $148 / 93$

\title{
Classical String Dynamics With NON-TRIVIAL TOPOLOGY
}

Thomas Deck

\begin{abstract}
The possibility of branching processes for classical strings is investigated on the basis of the Nambu-Goto action. We parametrize the world sheet by a Riemann surface $M$ and introduce a $C^{\infty}$-smooth, degenerate metric $\eta$ on $M$. Well-known results about the conformal group are generalized to the case of $(M, \eta)$. We provide a rigorous, infinite dimensional Hamiltonian setting for processes that change the topology of a string. Finally, the classical background for the theory of quantum strings as developed by Krichever and Novikov in 1987 is discussed within this classical framework.
\end{abstract}

Keywords: semi-Riemannian manifold, conformal symmetry, infinite dimensional Hamiltonian systems.

1991 AMS: $49 H 05,53 C 50,70 G 35,83 E 30$ 


\section{Introduction}

Classical string dynamics based on variational principles is a well-established theory $[1,2]$. For closed strings one usually considers string motions that are described by differentiable maps $X$ from $\mathbb{R} \times S^{1}$ to $\mathbb{R}^{d}$. The possibility of string splittings has been discussed in the context of cosmic strings [3,4] whereas this aspect has not been investigated in detail for classical fundamental strings. In the latter case the simple underlying idea is to treat a string which self-intersects as consisting of two strings that obey their own dynamics [5]. A consequence of such a viewpoint are. "light cone diagrams" and it has been shown by Giddings and Wolpert [6] that they provide a parametrization of the moduli space of compact Riemann surfaces. The problem of quantization of such diagrams has been tackled successfully by Krichever and Novikov $[7,8]$ and various aspects of that theory (called $K N$. theory) have been worked out since (cf. [9-13]). Nevertheless, the physical and geometric background on the classical level is still missing and we aim to fill that gap.

The paper is organized as follows: To fix notations, we recall some basic facts about closed strings in chapter one and we give an explicit solution for general string motions that allows us to study the geometry of world sheets in a direct way. The general solution is modified in the second chapter to processes where one string splits into two strings. From the principle of least action we derive a local criterion whether the branching solution is preferred or not.

Chapter three deals with the symmetry of topological non-trivial string world sheets. It turns out that the symmetry is conveniently described by a geometric structure on the "base manifold $M$ " that parametrizes the world sheet: This structure consists of a Riemann surface $M$ equipped with a smooth, degenerate semi-Riemannian metric $\eta$; those points $p_{c}$ on $M$ satisfying $\operatorname{det}\left(\eta\left(p_{c}\right)\right)=0$ correspond to branching points of the string motion. We then generalize results about conformal transformations of the manifold $\left(\mathbb{R} \times S^{1},-d \tau^{2}+d \sigma^{2}\right)$ to the case of $(M, \eta)$. Specificly we show that the conformal group is infinite dimensional and the associated conformal algebra splits into two commuting parts.

A Hamiltonian description that allows us to cope with the varying string topology is developed in chapter four: The classical phase space $P$ is substituted by a collection of spaces $\mathcal{P}=\bigcup_{\tau \in \mathbb{R}} P_{\tau}$ and a string motion is represented by a section $s: \mathbb{R} \rightarrow \bigcup_{\tau \in \mathbb{R}} P_{\tau}$, i.e. $s(\tau) \in P_{\tau}$. In order to obtain a well-defined motion for a specific initial string state, one must choose the Riemann surface properly. In this way the moduli space of compact Riemann surfaces appears naturally in the Hamiltonian setting. Next we investigate conserved quantities $Q_{\xi}$ having their origin in the algebra of conformal vector fields $\xi$; these quantities define (as usual) a realization of the conformal algebra on phase space. Moreover, we evaluate Poisson brackets of functions $Q_{\xi}$ with arbitrary fields $\xi$ on $M$, using their "conformal extension " $\xi_{c}$.

This method is applied to holomorphic vector fields in the last chapter: It results in a "local Wick rotation" on the Riemann surface $M$ and as a consequence the algebra of holomorphic vector fields on $M$ is also represented on phase space (this is not obvious because the theory possesses no Euclidean conformal symmetry). The paper concludes with the classical foundations of $\mathrm{KN}$-theory: They are described in a natural way within the formalism developed before. 


\section{Remarks on classical string dynamics}

Let us consider a classical closed string in Minkowski space ${ }^{* 1}\left(\mathbb{R}^{4}, g=\operatorname{diag}(-1,1,1,1)\right)$. Its world sheet $\Sigma$ is parametrized by functions $X^{\mu}\left(\sigma^{1}, \sigma^{2}\right), \mu=0, \ldots, 3$, with local $\left(\sigma^{1}, \sigma^{2}\right)$ parameters. String dynamics can be defined by an action $S$ which is proportional to the area of $\Sigma$. We choose the constant of proportionality equal minus one,

$$
S=\int L(X, \alpha) d^{2} \sigma=-\int \sqrt{-\operatorname{det}\left(h_{\alpha \beta}\right)} d^{2} \sigma
$$

where $h_{\alpha \beta}=\partial_{\alpha} X^{\mu} \partial_{\beta} X^{\nu} g_{\mu \nu} \equiv X, \alpha X,_{\beta}$ (spacetime contractions will be suppressed) denotes the induced metric on $\Sigma$. The Euler-Lagrange equations $\partial_{\alpha} \frac{\partial L}{\partial X, \alpha}=0$ are given by

$$
\square_{\text {cov }} X^{\mu}=h^{\alpha \beta} \nabla_{\alpha} \nabla_{\beta} X^{\mu}=0
$$

where $\square_{\text {cov }}$ labels the covariant d'Alembert operator. Notice that this equation is only welldefined if $\left(h_{\alpha \beta}\right)$ is non-degenerate. The physical meaning of $\operatorname{det}\left(h_{\alpha \beta}\right)=0$ can be recovered with conformal parameters $(\tau, \sigma)$ in which $h$ reads $(\Lambda \geq 0$ is a real valued function)

$$
h(\tau, \sigma)=\Lambda(\tau, \sigma) \cdot \eta \quad, \quad \eta=-d \tau^{2}+d \sigma^{2}
$$

We will construct such a conformal flat metric below (cf. [1],[14]). A first step is to choose the $\tau$-parameter to be proportional to proper time

$$
t=X^{0}(\tau, \sigma)=R \cdot \tau, \quad R=\text { const. }>0,
$$

and $\sigma, R$ will be specified shortly; as usual we set $\dot{X}^{\mu}=\frac{\partial X^{\mu}}{\partial \tau}, X^{\prime \mu}=\frac{\partial X^{\mu}}{\partial \sigma}$. Suppose that we have conformal parameters, then the metric $\Lambda \cdot \eta_{\alpha \beta} \stackrel{\partial \tau}{=} X, \alpha X,{ }_{, \beta}$ gives $\Lambda=-\dot{X}^{2}$ for $\alpha=\beta=0$ and (3) is equivalent to

$$
\dot{X}^{2}+X^{\prime 2}=0 \quad, \quad \dot{X} X^{\prime}=0
$$

$\dot{\vec{X}}:=\left(\dot{X}^{1}, \dot{X}^{2}, \dot{X}^{3}\right)$ is proportional to the velocity of the curve $t \rightarrow \vec{X}\left(\frac{t}{R}, \sigma\right)$ which we call the curve of a point $\sigma$. From $\Lambda=-\dot{X}^{2}$ it follows that the induced metric $h(3)$ is singular at $(\tau, \sigma)$ if and only if the point $\sigma$ moves at the velocity of light, $c=1$.

Definition: A closed string at time $t=0$ has admissible initial data if its position $\vec{X}_{0}$ in $\mathbb{R}^{3}$ as well as its initial velocity $\vec{u}_{0}=\dot{\vec{X}}(0, \cdot)$ are parametrizable smoothly by the Euclidean length $l$; moreover the relation $\left|\vec{u}_{0}(l)\right|<R$ (with respect to the metric $\delta=\left.g\right|_{\mathbb{R}^{3}}$ ) holds for all values of $l, 0 \leq l \leq L$.

We choose $\vec{u}_{0}$ orthogonal to the string*2 $\frac{d \vec{X}_{0}}{d l} \cdot \vec{u}_{0}=0$, and because $t$ does not depend on $l$ (4) we obtain $\frac{d X_{0}}{d l} u_{0}=0$. In order to get a conformal flat metric at least at $\tau=0$,

\footnotetext{
*1 The results on the classical level do not depend significantly on the dimension $d \geq 4$.

*2 This is not a dynamical restriction because the "infinitesimal surface" defined by the vectors $(R d \tau, \vec{u}(l) d \tau)$ coincides with the surface defined by $(R d \tau, \vec{u} \perp(l) d \tau)$ where $\vec{u}_{\perp}$ denotes that part of $\vec{u}$ which is orthogonal to the string.
} 
we must define the $\sigma$-parameter in such a way that $(5)$ is fulfilled, in particular we need $-R^{2}+\vec{u}_{0}^{2}+\vec{X}_{0}^{\prime 2}=0$. This enforces the definition $\left(d l=\sqrt{\vec{X}_{0}^{\prime 2}} d \sigma\right)$

$$
\sigma(l):=\frac{1}{R} \int_{0}^{l} \frac{1}{\sqrt{1-\vec{v}_{0}^{2}}} \dot{d l^{\prime}}
$$

where $\vec{v}_{0}=\left.\frac{\partial \vec{X}}{\partial t}\right|_{t=0}=\frac{\vec{u}_{0}}{R}$ is the physical velocity of the point $l(\sigma)$. The range of $\sigma$ becomes $[0,2 \pi]$ for $R:=\frac{1}{2 \pi} \int_{0}^{L} \frac{1}{\sqrt{1-\vec{v}_{0}^{2}}} d l$. The second part of $(5)$ now follows from $\frac{d X_{0}}{d l} u_{0}=0$, hence it is always possible to satisfy (5) at $\tau=0$. For $\tau>0$ we have:

Proposition 1.1 i) On $\mathbb{R} \times S^{1}$ the unique solution of $\square X^{\mu}=0$ with respect to $C^{2}$-smooth initial data $X^{\mu}(0, \sigma)=X_{0}^{\mu}(\sigma)$ and $\frac{\partial X^{\mu}}{\partial \tau}(0, \sigma)=u_{0}^{\mu}(\sigma)$ is given by

$$
X^{\mu}(\tau, \sigma)=\frac{1}{2}\left\{X_{0}^{\mu}(\sigma+\tau)+X_{0}^{\mu}(\sigma-\tau)\right\}+\frac{1}{2} \int_{\sigma-\tau}^{\sigma+\tau} u_{0}^{\mu}\left(\sigma^{\prime}\right) d \sigma^{\prime} \quad, \mu=0,1,2,3 .
$$

ii) The map $X: \mathbb{R} \times S^{1} \rightarrow \mathbb{R}^{4}$ defines a $C^{1}$-smooth metric $h=X^{*} g$ (possibly degenerate). on $M=\mathbb{R} \times S^{1}$. If the initial data obey (5) then $\square_{\text {cov }} X^{\mu}=0$ is satisfied at all $p \in M$ where $h(p)$ is non-degenerate.

Proof: i) is a standard fact. ii) With (6) one can directly verify (5) for all values of $\tau$, i.e. the pullback metric $h$ reads $h=\Lambda \cdot \eta$. In $(\tau, \sigma)$-parameters (which are coordinates on $\mathbb{R} \times S^{1}$ ) we obtain, together with (2)

$$
\square_{c o v} X^{\mu}=\partial_{\alpha} \frac{\partial L}{\partial X_{, \alpha}^{\mu}}=\partial_{\alpha}\left(\sqrt{h} h^{\alpha \beta} \partial_{\beta} X^{\mu}\right)=\partial_{\alpha}\left(\Lambda \frac{1}{\Lambda} \eta^{\alpha \beta} \partial_{\beta} X^{\mu}\right)
$$

In the case $\Lambda(\tau, \sigma) \neq 0$ this equation reduces to $\square_{\text {cov }} X^{\mu}=\partial_{\alpha}\left(\eta^{\alpha \beta} \partial_{\beta} X^{\mu}\right)=\square X^{\mu}=0$.

Remarks: 1 . Because (5) can always be satisfied at $\tau=0$ this proof shows the existence of global conformal parameters for on-shell string world sheets of closed strings. Notice that solution (6) for $X_{0}^{0}(\sigma)=0, u_{0}^{0}(\sigma)=R$ is given by (4).

2. Equation (7) reveals another useful property of conformal parameters: They allow us to regularize the equations of motion (2) at critical points $p_{c}$ where $h$ degenerates. But one should bear in mind that $\square X\left(p_{c}\right)=0$ is not derived rigorously from the variational principle.

A simple consequence of (6) is a property which is usually assumed to be valid:

Corollary 1.2 If a string has admissible initial data at $\tau=0$ then no point of the string will exceed the velocity of light for all later times $t>0$, i.e. we have $\left|\frac{\partial \vec{X}}{\partial t}(t, \sigma)\right| \leq 1$.

Proof: With (5) we obtain $d l(\sigma \pm \tau)=\sqrt{\vec{X}_{0}^{\prime 2}(\sigma \pm \tau)} d \sigma=R \sqrt{1-\vec{v}_{0}^{2}(\sigma \pm \tau)} d \sigma$. This yields

$$
\begin{aligned}
\dot{\vec{X}}(\tau, \sigma) & =\frac{R}{2}\left\{\frac{d \vec{X}_{0}(\sigma+\tau)}{d l} \sqrt{1-\vec{v}_{0}^{2}(\sigma+\tau)}+\vec{v}_{0}(\sigma+\tau)\right\} \\
& -\frac{R}{2}\left\{\frac{d \vec{X}_{0}(\sigma-\tau)}{d l} \sqrt{1-\vec{v}_{0}^{2}(\sigma-\tau)}-\vec{v}_{0}(\sigma-\tau)\right\}
\end{aligned}
$$


Hence, applying the triangle inequality in $\mathbb{R}^{3}$ we obtain (using $\frac{d \vec{X}_{0}}{d l} \cdot \vec{v}_{0}=0$ and $\left|\frac{d \vec{X}_{0}}{d l}\right|=1$ ):

$$
\left|\frac{\partial \vec{X}}{\partial t}(t, \sigma)\right| \leq \frac{1}{2} \sqrt{\left(1-\vec{v}_{0}^{2}(\sigma+\tau)\right)+\vec{v}_{0}^{2}(\sigma+\tau)}+\frac{1}{2} \sqrt{\left(1-\vec{v}_{0}^{2}(\sigma-\tau)\right)+\vec{v}_{0}^{2}(\sigma-\tau)}=1
$$

For $\dot{X}^{2}(p) \neq 0$ the map $X$ defines a local embedding of a neighbourhood $U(p) \subset \mathbb{R} \times S^{1}$ into $\mathbb{R}^{4}$, but when a point $\sigma$ reaches the velocity of light, the singular behaviour of the equations of motion is reflected by a singular behaviour of the world sheet $\Sigma$ : The unit tangent vector $\frac{\partial \vec{X}}{\partial !}=\frac{1}{\sqrt{-\dot{X}^{2}}} \frac{\partial \vec{X}}{\partial \sigma}$ may behave discontinuously only if $\dot{X}^{2}$ vanishes.

Special case: It is geometrically intuitive to see how a string with velocity zero at $\tau=0$ evolves. The parameter $\sigma=\frac{2 \pi}{L} \cdot l$ is proportional to the Euclidean length of the string and the solution $\vec{X}(\tau, \sigma)=\frac{1}{2}\left\{\vec{X}_{0}(\sigma+\tau)+\vec{X}_{0}(\sigma-\tau)\right\}$ has the following interpretation: After time $\tau$ the point $\vec{X}(\sigma, 0)=\vec{X}_{0}(\sigma)$ moved exactly to the middle between the points $\vec{X}_{0}(\sigma+\tau)$ and $\vec{X}_{0}(\sigma-\tau)$. The physical velocity is given by $\vec{v}(\tau, \sigma)=\frac{1}{2}\left\{\frac{d \vec{X}_{0}}{d l}(\sigma+\tau)-\frac{d \vec{X}_{0}}{d l}(\sigma-\tau)\right\}$. This gives a unit vector, i.e. the velocity of light, if and only if the tangent vectors $\frac{d \vec{X}_{0}}{d l}$ at the points $\vec{X}_{0}(\sigma+\tau)$ and $\vec{X}_{0}(\sigma-\tau)$ are parallel. Indeed, if the string lies in a two dimensional plane each point $\vec{X}(\sigma, 0)$ necessarily reaches the velocity of light because the angle $\phi(\tau)$ between the two tangent vectors $\frac{d \vec{X}_{0}}{d l}(\sigma+\tau)$ and $-\frac{d \vec{X}_{0}}{d l}(\sigma-\tau)$ varies continuously from $-\pi$ to $\pi$. The shape of a string in a neighbourhood of a point $\vec{X}\left(\sigma_{0}, \tau_{0}\right)$ with $|\vec{v}|=1$ can easily be derived by approximating the string at $\tau=0$ with parts of circles. One finds a cusp at $\vec{X}\left(\sigma_{0}, \tau_{0}\right)$ and this cusp generally exists within a whole time interval $\Delta \tau$, defining a cusp curve $c(\tau)$ in Euclidean $\mathbb{R}^{3}$. Examples of that behaviour are pictured in ref. [3].

\section{Modified string dynamics}

From (6) follows that the state of a string is reproduced after a period $\Delta t=R \cdot \pi$ up to a rigid translation $\Delta \vec{X}=\int_{-\pi}^{\pi} \vec{u}\left(\sigma^{\prime}\right) d \sigma^{\prime}$ and up to a parameter change $\sigma \rightarrow \sigma+\pi$. If the string doesn't self-intersect and if no point reaches $|\vec{v}|=1$ during one period then string dynamics is uniquely fixed and we have a global embedding of $\mathbb{R} \times S^{1}$ into $\mathbb{R}^{4}$. Now suppose that two branches of the string intersect, $\vec{X}\left(0, \tau_{0}\right)=\vec{X}\left(\sigma_{0}, \tau_{0}\right)$, having velocities $|\vec{v}|<1$ at the intersection point. The general configuration will be as shown in fig. 1 :

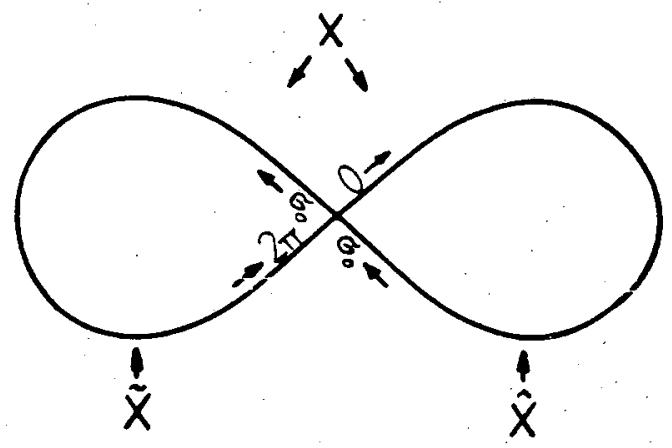

Fig.1 Intersecting string branches. 
Solution (6) for $X(\tau, \sigma)$ implies that these two branches move through each other without any influence. However, we might take a different point of view; namely that we are given two strings at $\tau_{0}$ each of which obeys its own dynamics for $\tau \geq \tau_{0}$ :

$$
\begin{gathered}
\widehat{X}(\tau, \sigma)=\frac{1}{2}\left\{Y\left(\sigma+\tau-\tau_{0}\right)+Y\left(\sigma-\tau+\tau_{0}\right)+\int_{\sigma-\tau+\tau_{0}}^{\sigma+\tau-\tau_{0}} r\left(\sigma^{\prime}\right) d \sigma^{\prime}\right\} \quad \sigma \in\left[0, \sigma_{0}\right) \\
\tilde{X}(\tau, \sigma)=\frac{1}{2}\left\{Z\left(\sigma+\tau-\tau_{0}\right)+Z\left(\sigma-\tau+\tau_{0}\right)+\int_{\sigma-\tau+\tau_{0}}^{\sigma+\tau-\tau_{0}} s\left(\sigma^{\prime}\right) d \sigma^{\prime}\right\} \quad \sigma \in\left[\sigma_{0}, 2 \pi\right) .
\end{gathered}
$$

Here the functions $Y(\sigma)=X\left(\sigma, \tau_{0}\right)$ and $r(\sigma)=\dot{X}\left(\sigma, \tau_{0}\right)$ have periodicity $\sigma_{0}$ and analogously $Z$ and $s$ are $\left(2 \pi-\sigma_{0}\right)$-periodic functions. The equations $\square \widehat{X}=\square \widetilde{X}=0$ are valid in a distributional sense because of the kinks which arise from the intersection point. We remark that no conservation law is violated by the splitting because all these conserved quantities are given by integrals that only contain first derivatives of the $X^{\mu}$. Of course energy, momentum and angular momentum behave additively under such a splitting. In consequence there is no obvious reason to exclude this alternative motion so let us consider how the action behaves as a function of time $\left(\tau_{0}:=0\right.$ for convenience). From (1) and (5) we obtain the on-shell action $\widetilde{S}=\int \dot{X}^{2} d^{2} \sigma$. We set $S_{\text {free }}(\tau):=\int_{0}^{\tau} \int_{0}^{2 \pi} \dot{X}^{2} d \sigma d \tau^{\prime}$, $S_{\text {split }}(\tau)=\int_{0}^{\tau} \int_{0}^{\sigma_{0}} \dot{\widehat{X}}^{2} d \sigma d \tau^{\prime}+\int_{0}^{\tau} \int_{\sigma_{0}}^{2 \pi} \dot{\widetilde{X}}^{2} d \sigma d \tau^{\prime}$ and examine the difference of the two areas.

Proposition 2.1 The frst and second derivative of $\Delta S(\tau):=S_{\text {free }}(\tau)-S_{s p l i t}(\tau)$ at $\tau=0$ is given by $\Delta \dot{S}(0)=0$ and

$$
\frac{d^{2} \Delta S}{d \tau^{2}}(0)=\left(\vec{u}_{0}\left(\sigma_{0}\right)-\vec{u}_{0}(0)\right)^{2}-\left(\vec{X}_{0}^{\prime}\left(\sigma_{0}\right)-\vec{X}_{0}^{\prime}(0)\right)^{2}
$$

Proof: The following formula is an immediate consequence of the fact that the values $X^{\mu}(\tau, \sigma)$ for both kinds of solutions coincide outside the $\sigma$-intervals $[-\tau, \tau]$ and $\left[\sigma_{0}-\tau, \sigma_{0}+\tau\right]$

$$
\begin{aligned}
\frac{d \Delta S}{d \tau}(\tau) & =\int_{-\tau}^{\tau}\left[\dot{X}^{2}(\tau, \sigma)+\dot{X}^{2}\left(\tau, \sigma_{0}+\sigma\right)\right] d \sigma \\
& -\int_{0}^{\tau}\left[\dot{\widehat{X}}^{2}(\tau, \sigma)+\dot{\widehat{X}}^{2}\left(\tau, \sigma_{0}-\tau+\sigma\right)\right] d \sigma-\int_{-\tau}^{0}\left[\dot{\tilde{X}}^{2}(\tau, \sigma)+\dot{\widetilde{X}}^{2}\left(\tau, \sigma_{0}+\tau+\sigma\right)\right] d \sigma .
\end{aligned}
$$

$\Delta \dot{S}(0)=0$ is now obvious. One must be cautious with derivatives of terms like $\int_{0}^{\tau} \dot{\widehat{X}}^{2}(\tau, \sigma) d \sigma$ because $\dot{\hat{X}}^{2}(\tau, \sigma)$ is discontinuous at $\sigma=\tau$. But it is standard analysis to show $\left.\frac{d}{d \tau}\right|_{\tau=0} \int_{0}^{\tau} \dot{\hat{X}}^{2}(\tau, \sigma) d \sigma=\lim _{\epsilon \downarrow 0} \dot{\hat{X}}^{2}(\epsilon, 0)$. Differentiating $\Delta \dot{S}(\tau)$ at $\tau=0$ and collecting terms that come from $(6),(8)$ and $(9)$ yields $\Delta \ddot{S}(0)=\left(u_{0}\left(\sigma_{0}\right)-u_{0}(0)\right)^{2}-\left(X_{0}^{\prime}\left(\sigma_{0}\right)-X_{0}^{\prime}(0)\right)^{2}$. Since the time component of this expression vanishes we obtain (10). 
We note from $\Delta \dot{S}(0)=0$ that at $\tau=0$ the two surfaces grow at the same rate at first order. If $\Delta \ddot{S}(0)$ is positive, the value $S_{\text {free }}(\tau)$ is greater than $S_{s p l i t}(\tau)$ within some time interval $[0, \epsilon]$. One may now modify string dynamics by the requirement of least growth of $S$ which then implies splitting and merging effects of strings on a classical level. The term $\left(\vec{u}\left(\sigma_{0}\right)-\vec{u}(0)\right)^{2}$ can be interpreted as follows: At high relative velocities of the two string branches the splitting solution is preferred whereas at low relative velocities these two branches do not interact. To see what the second term means, suppose that the string is at rest $\left(\vec{u}_{0}=0\right)$ and we treat the two string branches locally as straight lines. Then after time $\tau$ there are two strings, each of which has two kinks and all points $\vec{X}(\tau, \sigma)$ between such two kinks exactly move at the speed $\frac{1}{2}\left|\vec{X}_{0}^{\prime}\left(\sigma_{0}\right)-\vec{X}_{0}^{\prime}(0)\right|$ (so by this effect the resulting action is increased).

Remark: This dynamic is not invariant under time reversal: Suppose we have a splitting process at $\tau=0$. Then the time reversed motion is a process where two strings merge into one. However, the minimality criterion forces us to take also a splitting solution for $\tau<0$, because at $\tau=0$ only $\vec{u}_{0}$ changes to $-\vec{u}_{0}$ which has no effect on (10).

From the last chapter we know that the situation is different when a point $\sigma_{0}$ of the string reaches the velocity of light (at $\tau_{0}$ ). If we express the equations of motion in the parameters $(\tau, l)$ instead of $(\tau, \sigma)$ we find that the tangential part of the string acceleration becomes singular at $\vec{X}\left(\sigma_{0}, \tau_{0}\right)$. From this intrinsic point of view (in contrast to the variational viewpoint) it seems to be natural to consider string branchings at such points. Geometrically it means that two cusp curves intersect at the same time and examples can be constructed by hand, using the "special case" discussed in chapter 1. A splitting solution is given (as for $\left|\vec{v}\left(\sigma_{0}, \tau_{0}\right)\right|<1$ ) by equations $(8,9)$. The main difference here is the fact that for both the classical as well as the splitting solution the equations of motion are not well-defined at the critical point because $h\left(\tau_{0}, \sigma_{0}\right)=0$. Outside this point both solutions are well-defined, hence there is no natural preference of one of them. This is somewhat unsatisfying but similar (non unique) situations also arise in classical one dimensional mechanics $^{* 3}$. One might argue that $\square X=0$ is only valid in a distributional sense and that it is unnatural for a string to move with kinks*4. But even this argument against string branchings can be refuted because of

Lemma 2.2: Suppose we have a string at $\tau=0$ with admissible initial data and at $\tau_{0}$ there is a string splitting with parallel velocity vectors $\vec{v}\left(\tau_{0}, 0\right)=\vec{v}\left(\tau_{0}, \sigma_{0}\right)$ at the speed of light. Then both strings (for $\tau>\tau_{0}$ ) are described by $C^{1}$-functions.

*3 A point particle in the $C^{1}$-potential $U(x)=-\frac{1}{2}|x|^{\frac{4}{3}}$ with position $x(0)=0$ and velocity $\dot{x}(0)=0$ has the choice to stay at the origin $(x(t)=0)$, or to move away $\left(x(t)=\left(\frac{t}{3}\right)^{3}\right)$ : Both motions obey $\ddot{x}(t)=-\frac{d U}{d x}(x(t))$.

*4 For cosmic strings there are good arguments for this viewpoint: Finite-width corrections to the Nambu action of Nielsen-Olesen strings involve a curvature term which suppresses kinks, see [15]. 
Proof: The one-sided derivatives of $(8)$ are given by

$$
\frac{\partial \widehat{X}}{\partial \sigma}(\tau, \sigma)=\frac{1}{2}\left\{X_{0}^{\prime}(\sigma+\tau)+X_{0}^{\prime}(\sigma-\tau)+u(\sigma+\tau)-u(\sigma-\tau)\right\} \quad, \quad \sigma=\sigma \bmod \sigma_{0}
$$

and the last two terms are continuous due to our assumption. Discontinuities can only arise at $\sigma=\tau$ or $\dot{\sigma}=\sigma_{0}-\tau$ because only then one argument of $X_{0}^{\prime}$ takes a value at which the splitting occurred. From $\dot{X}^{2}(0,0)=\dot{X}^{2}\left(0, \sigma_{0}\right)=0$ and $\dot{X}^{2}+X^{\prime 2}=0$ we obtain $\vec{X}_{0}^{\prime}(0)=\vec{X}_{0}^{\prime}\left(\sigma_{0}\right)=0$ so left-sided and right-sided derivatives of $\widehat{X}$ coincide. The same arguments hold for the $\tau$-derivative which completes the proof.

In consequence, no kinks emerge from the intersection point and $\square X=0$ is obeyed in an ordinary sense. Notice that we have $\Delta \ddot{S}(0)=0(10)$, so the case with parallel light vectors is rather distinguished. So far we discussed splitting solutions of the form $(8,9)$ but it is now obvious how to construct merging solutions of the same type. The only difference would be that we have to perform some shifts $\sigma_{i} \rightarrow \sigma_{i}+\Theta_{i}$ along each string in order to get a continuous parametrization of that merging solution.

Remark: We argue that a closed string cannot split into an open string, even at points $\sigma_{0}$ that move at the velocity of light. The boundary conditions for open strings impose constraints on the Fourier modes of such a string. If these constraints are satisfied for the state $X_{0}$ of a closed string then we can interpret $X_{0}$ as initial state for an open string. We now alter the state $X_{0}$ outside a neighbourhood of $\sigma_{0}$ such that the constraints fail for the new state $\widetilde{X}_{0}$, hence $\widetilde{X}_{0}$ cannot describe the initial configuration of an open string. On the other hand suppose that there is a criterion which allows $X_{0}$ to split. This criterion must be of a local nature (like Proposition 2.1) in order to obtain a local interaction. Consequently the state $\widetilde{X}_{0}$ is also allowed to split which is an obvious contradiction.

Now consider the history of $n$ classical strings which do not interact at times $\tau<\tau_{1}$, i.e. we have $n$ incoming strings. We restrict the discussion to the case of finitely many string branchings that occur at times $\tau_{1}, . ., \tau_{k}$. The resulting configuration consists of $m$ strings for $\tau>\tau_{k}$, i.e. $m$ outgoing strings. In ref. [6] it has been shown that such a process (which was treated on the formal level of a diagram) can be parametrized by a Riemann surface $M$. The position of the strings on $M$ at a fixed moment of time $\tau_{0}$ is given by

$$
C_{\tau_{0}}=\left\{q \in \dot{M} \mid \tau(q)=\operatorname{Re} \int_{q_{0}}^{q} d k=\tau_{0}\right\}
$$

where $q_{0}$ is some fixed reference point and $d k$ is a unique meromorphic differential on a compact Riemann surface $\bar{M} \supset M$ that has simple poles at the points $P_{i}, i=1, . ., n$ and $Q_{j}, j=1, . ., m$ and is holomorphic elsewhere $\left(M=\bar{M} \backslash\left\{P_{1}, . ., P_{n}, Q_{1}, . ., Q_{m}\right\}\right)$. At the $P_{i}$ (corresponding to $\tau=-\infty$ ) $d k$ has real positive residues whereas at the $Q_{j}$ (corresponding to $\tau=+\infty$ ) there are only real negative residues. The critical points of the harmonic function $\tau: M \rightarrow \mathbb{R}$ are given by the zeros of $d k$ and these points coincide with those $p_{c}$ where the strings split or rejoin. We denote a critical value of $\tau$ by $\tau_{c}: \tau_{c} \in\left\{\tau_{1}, . ., \tau_{k}\right\}$. In conformal $(\tau, \sigma)$-parameters $d k$ has the simple form $d k=d \tau+i d \sigma$ (indeed, this is the definition of $d k$ given in [6]). The $\sigma$-parameter has the property to be defined modulo some angle $\sigma_{i}$ along each string and $\sigma$ may change discontinuously by some twist angle $\Theta$ if two 
strings merge. This behaviour is exactly described by $\sigma=\operatorname{Im} \int_{q_{0}}^{q} d k$ because $d k$ has pure imaginary periods. Summarizing, we end up with the following situation:

A parameter surface of the string world sheet can always be described by a smooth submanifold $M$ of a compact Riemann surface $\bar{M}$ together with a unique meromorphic differential $d k$ on $\bar{M}$; we call $(M, d k)$ base manifold. If a point $q_{0} \in M$ is not a critical point then the parameters $u=\tau+i \sigma=\int_{q_{0}}^{q} d k$ define a local holomorphic chart around $q_{0}$. A crucial observation is that these charts consist of conformal parameters for the string world sheet.

\section{Conformal symmetry}

The natural geometric structure on a base manifold $(M, d k)$ associated to a specific string motion $X: M \rightarrow \mathbb{R}^{4}$ is given by the pullback $X^{*} g=: h$ of the metric $g$ in Minkowski space. In general, the world sheet $\Sigma=X(M)$ is not a smooth submanifold of $\mathbb{R}^{4}$, even in the simplest case of $M=\mathbb{R} \times S^{1}$. Only if $X$ is an embedding we can identify $M$ with $X(M)$ and in that case the "induced metric h on $\Sigma$ " is well-defined. Hence for an arbitrary $C^{1}$-string motion there is a $C^{0}$-metric ${ }^{* 5}$ on $M$, whereas $X(M)$ doesn't carry a geometric structure. This is one reason why $M$, not $X(M)$, is relevant in the subsequent considerations. Another reason is even more compelling: In a conformal chart $V \subset M^{\circ}:=M \backslash\left\{\right.$ critical points $\left.p_{c}\right\}$ the string action (1) can be written as

$$
\widetilde{S}_{V}=\int_{V} \dot{X}^{2} d^{2} \sigma=-\frac{1}{2} \int_{V} \eta^{\alpha \beta} \partial_{\alpha} X \partial_{\beta} X d^{2} \sigma
$$

where we used (5). $\widetilde{S}_{V}$ is locally (i.e. for any domain $V \subset \dot{M}$ ) invariant under conformal diffeomorphisms $\widetilde{\Psi}: V \rightarrow V^{\prime} \subset \dot{M}$, satisfying $\widetilde{\Psi}^{*} \eta=F \cdot \eta$ with a smooth function $F$. This observation is crucial with respect to the geometry of $\dot{M}^{\circ}$ because it follows that the metric $\eta$ is of physical importance and not the pullback metric $-\dot{X}^{2} \cdot \eta$, which can be discontinuous and degenerate on $\dot{M}^{\circ}$.

Let $\left\{\widetilde{\Psi}_{s}, s \in \mathbb{R}\right\}$ be a conformal flow on $\dot{M}$, i.e. a one parameter group of diffeomorphisms, where each $\widetilde{\Psi}_{s}$ is a conformal transformation from $\dot{M}^{\circ}$ to $\mathscr{M}$. We extend $\widetilde{\Psi}_{s}$ to a map $\Psi_{s}: M \rightarrow M$ by the definition $\Psi_{s}\left(p_{c}\right)=p_{c}$ and we assume that $\Psi_{s}$ is a smooth map (cf. the remark following Corollary 4.5). This situation can be described globally on $M$ if the definition $\eta\left(p_{c}\right)=0$ makes sense because then the critical points $p_{c}$ are necessarily fixed points of any conformal flow $\Psi_{s}$ on $(M, \eta)$.

Lemma 3.1 The metric $\eta=-d \tau^{2}+d \sigma^{2}$ on $M^{\circ}$ has a unique $C^{\infty}$-smooth extension to $M$ which is given by $\eta\left(p_{c}\right)=0$ for all critical points $p_{c}$ on $M$.

Proof: We represent $\eta$ in a chart around $p_{c}$ with coordinates $\left({\sigma^{0}}^{\prime}, \sigma^{1^{\prime}}\right)=\left({\tau^{\prime}}^{\prime}, \sigma^{\prime}\right), p_{c} \simeq(0,0)$. The parameter transformation is of the form (cf. [6]) $\tau+i \sigma-a=\left(\tau^{\prime}+i \sigma^{\prime}\right)^{n}$ with $n \geq 2$ and some constant $a$. Therefore the matrix $\frac{\partial \sigma^{\alpha}}{\partial \sigma^{\alpha^{\prime}}}$ depends analytically on $\tau^{\prime}, \sigma^{\prime}$ and vanishes in the limit $p \rightarrow p_{c}$. From $\eta_{\alpha^{\prime} \beta^{\prime}}=\frac{\partial \sigma^{\alpha}}{\partial \sigma^{\alpha^{\prime}}} \frac{\partial \sigma^{\beta}}{\partial \sigma^{\beta^{\prime}}} \eta_{\alpha \beta}$ we obtain the required statement.

\footnotetext{
*5 Strings with kinks give rise to metrics h that are discontinuous along those trajectories on $M$ which parametrize the kinks.
} 
Notice that the definition of $\eta$ is independent of the special string process under consideration but it fits well into the picture of string motion in the case $\dot{X}^{2}\left(p_{c}\right)=0$ because then the induced metric $h=-\dot{X}^{2} \cdot \eta$ vanishes in $(\tau, \sigma)$-coordinates in the limit $p \rightarrow p_{c}$.

Definition: The conformal group of $(M, \eta)$ consists of all diffeomorphisms $\Psi: M \rightarrow M$ which obey $\Psi^{*} \eta=\Omega \cdot \eta$, where $\Omega \geq 0$ denotes a smooth function on $M$.

We are now prepared to study conformal vector fields $\xi=\dot{\Psi}_{0}$ which generate a conformal flow $\Psi_{s}$ on $M$. Obviously we have $\xi\left(p_{c}\right)=\dot{\Psi}_{0}\left(p_{c}\right)=0$. The Lie algebra of conformal vector fields on $(M, \eta)$ is denoted by $\mathcal{A}_{M}$. It is well-known that in $(\tau, \sigma)$-parameters $\xi=\xi^{0} \partial_{\tau}+\xi^{1} \partial_{\sigma} \in \mathcal{A}_{M}$ has to obey the "pseudo" Cauchy-Riemann equations

$$
\xi, 0, \xi_{, 1}^{1} \quad, \quad \xi_{, 0}^{1}=\xi_{, 1}^{0} .
$$

The general solution of (13) in local light-cone coordinates $\sigma^{ \pm}$reads $\xi=f\left(\sigma^{+}\right) \partial_{\sigma^{+}}+$ $g\left(\sigma^{-}\right) \partial_{\sigma^{-}}$, with $\sigma^{ \pm}=\tau \pm \sigma$ and $\partial_{\sigma^{ \pm}}=\frac{1}{2}\left(\partial_{\tau} \pm \partial_{\sigma}\right)$. From the critical points $p_{c}$ we do not receive any constraints upon the derivatives of $\xi$ because the resulting constraint equations are trivial $(0=0)$ due to the vanishing of $\eta$ at $p_{c}$. Of course, the local decomposition of $\xi$ defines a unique global decomposition $\xi=\xi^{+}+\xi^{-}$with Lie bracket $\left[\xi^{+}, \xi^{-}\right]=0$.

Theorem 3.2 $\mathcal{A}_{M}$ is the direct sum of two commuting infinite dimensional subalgebras, $\mathcal{A}_{M}=\mathcal{A}_{M}^{+} \oplus \mathcal{A}_{M}^{-}$. Each $\xi=\xi^{+}+\xi^{-} \in \mathcal{A}_{M}$ is uniquely determined by its restriction along an arbitrary but fixed time slice $C_{\tau}$ (11). It can be constructed via parallel translation of $\left.\xi^{ \pm}\right|_{C_{\tau}}$ along light curves $\gamma^{ \pm}$which obey $\sigma^{ \pm}=$const. in local light-cone coordinates.

Proof: In a neighbourhood of any point $p \neq p_{c}$ we have $\xi=f\left(\sigma^{+}\right) \partial_{\sigma^{+}}+g\left(\sigma^{-}\right) \partial_{\sigma^{-}}$and the coefficients $f\left(\sigma^{+}\right)$and $g\left(\sigma^{-}\right)$are constant along the curves $\gamma^{+}$and $\gamma^{-}$respectively. Hence $\xi^{+}$and $\xi^{-}$coincide with those fields which arise from $\left.\xi^{ \pm}\right|_{C_{\tau}}$ via parallel transport along $\gamma^{ \pm}$(with respect to the metric $\eta$ ). On a curve $\gamma_{c}^{+}$which meets a critical point $p_{c}$ the field $\xi^{+}$must vanish since otherwise $\xi\left(p_{c}\right)$ becomes singular $\left(\frac{\partial \sigma^{\alpha \prime}}{\partial \sigma^{\alpha}}\right.$ diverges at $\left.p_{c}\right)$. An analogous argument holds for $\gamma_{c}^{-}, \xi^{-}$. The curves $\gamma^{ \pm}$arising from $C_{\tau}$ cover $M$ in a unique way: Along a fixed "string tube" $\left[\tau_{1}, \tau_{2}\right] \times S^{1}$ these curves wind up with a constant slope of $\frac{\Delta \tau}{\Delta \sigma}=\mp 1$ (an example is pictured in fig.2). Therefore the components $\xi^{ \pm}$are fixed by this construction.

The zeros of $\xi^{ \pm}$along $\gamma^{ \pm}$impose finitely many vanishing conditions on $\left.\xi^{ \pm}\right|_{C_{r}}$, say at the points $p_{1}, . ., p_{n} \in C_{\tau}$. From a smooth function $f$ on $C_{\tau}$ that vanishes in some neighbourhood of $\left\{p_{1}, . ., p_{n}\right\}$ we receive the vectors $\left.f(\sigma) \partial_{\sigma^{+}}\right|_{C_{\tau}}$. They define a global conformal vector field $\xi^{+}$on $M$ via parallel translation along $\gamma^{+}$. Since the vector space of such functions $f$ on $C_{\tau}$ is infinite dimensional, so is $\mathcal{A}_{M}^{+}$and of course also $\mathcal{A}_{M}^{-}$.

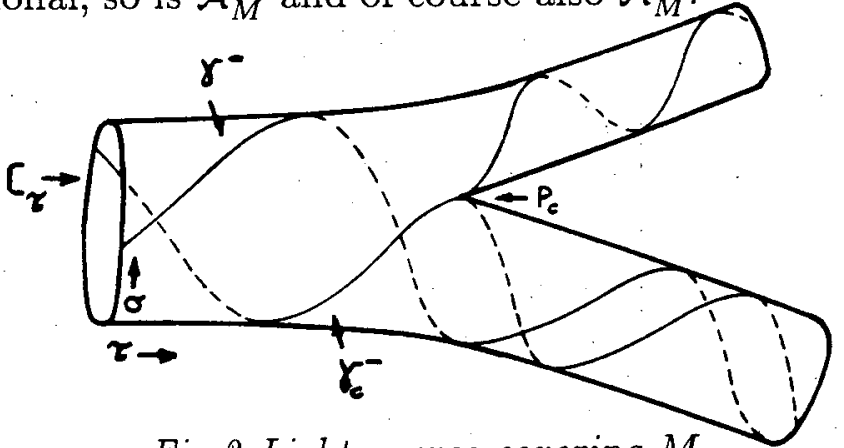

Fig.2 Light curves covering $M$. 
Thé $\xi^{ \pm}$-coefficients of $\xi=x i^{+} \partial_{\sigma^{+}}+\xi^{-} \partial_{\sigma^{-}}$are bounded on $C_{\tau}$ which implies

Theorem 3.3: The conformal group of $(M, \eta)$ is infinite dimensional.

Proof: It suffices to show that for each generator $\xi \in \mathcal{A}_{M}$ the conformal flow $\Psi_{s}=\exp (s \xi)$ exists on $M$ for all $s \in \mathbb{R}$. Remember that we denoted the set of critical values $\tau_{c}$ by $\left\{\tau_{1}, \ldots, \tau_{k}\right\}$. Each time slice $C_{\tau}$ with $\tau \in\left(\tau_{k}, \infty\right)$ consists of m components $C_{\tau}^{j}, j=1, . ., m$. The $C_{\tau}^{j}$ give rise to the subspace $M_{j}:=\bigcup_{\tau \in\left(\tau_{k}, \infty\right)} C_{\tau}^{j} \subset M$ that is parametrized by holomorphic coordinates $u=\tau+i \sigma$ with $\sigma=\sigma \bmod \sigma_{j}$. From $z=x+i y=e^{-\frac{2 \pi}{\sigma_{j}} u}$ we obtain another holomorphic coordinate and the value $z=0$ corresponds to a point $Q_{j}$ outside $^{* 6}$ of $M$. One verifies by direct computation that the components of $\xi$ represented in $(x, y)$-coordinates vanish in the limit $(x, y) \rightarrow 0$ and therefore we can extend continuously $\xi$ to $\bar{\xi}$ by the definition $\bar{\xi}\left(Q_{j}\right)=0, j=0, . ., m$. Analogously we set $\bar{\xi}\left(P_{i}\right)=0, i=0, . ., n$, for those points $P_{i}$ corresponding to the $n$ components of $C_{\tau}\left(\tau<\tau_{1}\right)$ for $\tau \rightarrow-\infty$. The continuous field $\bar{\xi}$ is defined on the compact manifold $\bar{M}$ so it generates a global flow $\left\{\bar{\Psi}_{s} \mid s \in \mathbb{R}\right\}$ on $\bar{M}$. The conformal flow on $M$ is now given by $\exp (s \xi)=\left.\bar{\Psi}_{s}\right|_{M}$.

Remark: Because of the vanishing constraints on $\left.\xi^{ \pm}\right|_{C_{\tau}}$ it is not possible to use an arbitrary smooth vector field e.g. $f\left(\sigma^{+}\right) \partial_{\sigma^{+}}$along $C_{\tau}$ to define $\xi^{+}$on $M$ via parallel translation: The result would be a vector field on $M$ which at certain points along the strings behaves like a step function and which is smooth outside these points. Such a vector field does not generate a diffeomorphism of $M$. This is the geometrical reason why the Virasoro generators $\xi_{n}^{+}=\left.e^{i n \sigma^{+}} \partial_{\sigma^{+}}\right|_{C_{\tau}}$ (for $C_{\tau} \simeq S^{1}$ ) do not define conformal transformations on the base manifolds $M$ pictured in fig.2. Nevertheless we can expand the restriction of a smooth conformal vector field $\xi^{+}$for $\tau<\tau_{1}$ in a series $\left.\xi^{+}\right|_{C_{\tau}}=\sum a_{n} e^{i n \sigma^{+}} \partial_{\sigma^{+}} \mid C_{\tau}$. The parallel translation of the fields $\left.e^{i n \sigma^{+}} \partial_{\sigma^{+}}\right|_{C_{\tau}}$ provides an expansion $\xi^{+}\left(\sigma^{+}\right) \partial_{\sigma^{+}}=\sum a_{n} e^{i n \sigma^{+}} \partial_{\sigma^{+}}$ on $M$ where the fields $\xi_{n}^{+}=e^{i n \sigma^{+}} \partial_{\sigma^{+}}$are discontinuous on $C_{\tau}$ for values $\tau>\tau_{1}$. Unfortunately several difficulties with Poisson brackets arise if we use such discontinuous vector fields in the Hamiltonian treatment of string dynamics. This will be explained in the next chapter.

\section{Hamiltonian description and conserved charges}

Due to Noether's theorem for each conformal vector field $\xi$ a conserved quantity (called charge) is associated:

$$
Q_{\xi}=\int_{C_{\tau}} \xi^{\alpha} T_{\alpha}^{0} d \sigma
$$

where $T$ denotes the canonical energy momentum tensor $T_{\alpha}{ }^{\beta}=X,{ }_{\alpha} X^{, \beta}-\frac{1}{2} \delta_{\alpha}^{\beta} X,{ }_{\gamma} X^{, \gamma}$ in $(\tau, \sigma)$-coordinates, derived from $\widetilde{S}$. Notice that $\eta$ degenerates at $p_{c}$, so there is no naturally induced isomorphism between $T_{p_{c}} M$ and $T_{p_{c}}^{*} M$. Hence we can only raise and lower indices outside $p_{c}$. Of course, $Q_{\xi}$ is conserved in time because $T_{\alpha}{ }^{\beta}$ vanishes on-shell.

*6 In this way the compactification $\bar{M}$ of $M$ is defined. 
Before we consider the Hamiltonian theory with varying string topology let us first set up the general context needed. The simplest Hamiltonian description for the classical string $\left(M=\mathbb{R} \times S^{1}\right)$ is to start with

$$
H(X, \Pi)=\frac{1}{2} \int_{S^{1}}\left(X^{\prime 2}+\Pi^{2}\right) d \sigma
$$

together with the constraints $\Pi X^{\prime}=0, \Pi^{2}+X^{\prime 2}=0$ imposed on the initial data of a string motion. Usually, a symplectic structure is fixed by setting $\left\{X^{\mu}(\sigma), \Pi^{\nu}\left(\sigma^{\prime}\right)\right\}=g^{\mu \nu} \delta\left(\sigma-\sigma^{\prime}\right)$. However the evaluation of $\left\{Q_{\xi}, Q_{\eta}\right\}$ is not possible for discontinuous vector fields $\xi$ and $\eta$ e.g. those which arise from Virasoro generators if $C_{\tau} \simeq S^{1}$, because then one gets products of delta functions. To clear up these problems within a rigorous setting (cf. [16]) we start with a phase space

$$
\left.P:=\left(\oplus_{\mu=0}^{3} H^{1}\left(S^{1}\right)\right) \oplus\left(\oplus_{\mu=0}^{3} L^{2} \dot{(} S^{1}\right)\right)
$$

which we denote by $H^{1}\left(S^{1}\right)^{4} \times L^{2}\left(S^{1}\right)^{4}$ for short. $H^{1}\left(S^{1}\right)$ stands for the first Sobolev space over $\mathbb{R}$. Note that $P$ is the natural domain of definition for the Hamiltonian $H$ and of course a Hilbert space with the scalar product

$$
<\left(X_{0}, \Pi_{0}\right),\left(X_{1}, \Pi_{1}\right)>_{P}:=\sum_{\mu=0}^{3}\left(<X_{0}^{\mu}, X_{1}^{\mu}>_{H^{1}}+<\Pi_{0}^{\mu}, \Pi_{1}^{\mu}>_{L^{2}}\right) .
$$

The canonical weak symplectic form $\omega: T P \times T P \rightarrow \mathbb{R}$ is defined by

$$
\omega_{(X, \Pi)}((\alpha, \beta) ;(\gamma, \delta)):=<\alpha^{\mu}, \delta_{\mu}>_{L^{2}}-<\gamma^{\mu}, \beta_{\mu}>_{L^{2}}
$$

with $(\alpha, \beta),(\gamma, \delta) \in T_{(X, \Pi)} P=H^{1}\left(S^{1}\right)^{4} \times L^{2}\left(S^{1}\right)^{4}$. Because $\omega$ is only a weak symplectic form the defining equation for Hamiltonian vector fields $X_{f}$, df $(\alpha, \beta)=$ $\omega\left(X_{f} ;(\alpha, \beta)\right) \forall(\alpha, \beta) \in T P$, need not have a solution at each $(X, \Pi) \in P$. In consequence the Poisson bracket of $C^{1}$-functions $f, g$ on $P,\{f, g\}:=\omega\left(X_{f} ; X_{g}\right)$, is only defined on the common domain $D=D_{f} \cap D_{g}$ of the fields $X_{f}$ and $X_{g}$.

Especially for the vector field $X_{H}=\left(\Pi, X^{\prime \prime}\right)$ we have $D_{H}=H^{2}\left(S^{1}\right)^{4} \times H^{1}\left(S^{1}\right)^{4}$. which is a dense subspace of $P$. The solution of the Hamiltonian equation of motion, $(\dot{X}, \dot{\Pi})=X_{H}$, is given by formula (6) together with its $\tau$-derivative $\dot{X}=\Pi$. It defines for each $\tau$ a continuous linear map $\widetilde{\Phi}_{\tau}:(X(0), \Pi(0)) \rightarrow(X(\tau), \Pi(\tau))$ on $H^{2}\left(S^{1}\right)^{4} \times H^{1}\left(S^{1}\right)^{4}$. This map has a unique continuous extension

$$
\Phi_{\tau}: P \rightarrow P,
$$

which gives the Hamiltonian flow of $X_{H}$ on $P$. In this way strings with kinks can naturally be described in the Hamiltonian setting.

We express the conserved quantity (14) in phase space variables. $Q_{\xi}: P \rightarrow \mathbb{R}$ is given by

$$
Q_{\xi}(X, \Pi)=-\int_{S^{1}}\left[\frac{1}{2} \xi^{0}\left(\Pi^{2}+X^{\prime 2}\right)+\xi^{1} \Pi X^{\prime}\right] d \sigma
$$


Of course $Q_{\xi}$ is also well-defined if we plug piecewise continuous functions $\xi^{0}$ and $\xi^{1}$ into (16). Then $Q_{\xi}$ is a $C^{\infty}$-smooth function on $P$ and its Fréchet derivative is given by

$$
d Q_{\xi}(X, \Pi)(\alpha, \beta)=-\int_{S^{1}}\left[\left(\xi^{0} X^{\prime}+\xi^{1} \Pi\right) \alpha^{\prime}+\left(\xi^{0} \Pi+\xi^{1} X^{\prime}\right) \beta\right] d \sigma \quad, \quad(\alpha, \beta) \in T_{(X, \Pi)} P .
$$

Lemma 4.1 Let $\xi^{0}$, $\xi^{1}$ be continuous functions on $S^{1}$ which are piecewise of class $C^{1}$. Then there exists a Hamiltonian vector field $X_{Q_{\xi}}$ with domain $D_{Q_{\xi}}=H^{2}\left(S^{1}\right)^{4} \times H^{1}\left(S^{1}\right)^{4}$ :

$$
X_{Q_{\xi}}=\left(Y_{\xi}, Z_{\xi}\right)=\left(-\xi^{0} \Pi-\xi^{1} X^{\prime},-\left(\xi^{0} X^{\prime}+\xi^{1} \Pi\right)^{\prime}\right)
$$

Proof: From the assumptions we obtain $\xi^{0} X^{\prime \mu}+\xi^{1} \Pi^{\mu} \in H^{1}\left(S^{1}\right)$ hence we can integrate (17) by parts and the defining equation for $X_{Q_{\xi}}$ yields:

$$
\int_{S^{1}}\left[\left(\xi^{0} X^{\prime}+\xi^{1} \Pi\right)^{\prime} \cdot \alpha-\left(\xi^{0} \Pi+\xi^{1} X^{\prime}\right) \cdot \beta\right] d \sigma=<Y_{\xi}, \beta>_{L^{2}}-<Z_{\xi}, \alpha>_{L^{2}}
$$

Notice that the domain of $X_{Q_{\xi}}$ coincides with those of $X_{H}=\left(\Pi, X^{\prime \prime}\right)$.

Remark: As mentioned in the last chapter we are also interested in the case where $\xi^{0}$ and $\xi^{1}$ stem from discontinuous vector fields $\xi=\left.\xi^{+}\right|_{C_{\tau}}+\left.\xi^{-}\right|_{C_{\tau}}$ with $C_{\tau}=\{\tau\} \times S^{1}$. Therefore we have to find $X_{Q_{\xi}}$ together with its domain $D_{Q_{\xi}} \subset H^{2}\left(S^{1}\right)^{4} \times H^{1}\left(S^{1}\right)^{4}$. It is a standard fact that for $L^{2}\left(S^{1}\right)$-functions $f, g$ and $\alpha^{\prime}$ the equation $\int_{S^{1}} g \alpha^{\prime} d \sigma=-\int_{S^{1}} f \alpha d \sigma$ is fulfilled if and only if $g \in H^{1}\left(S^{1}\right)$ and then $f=g^{\prime}$ holds. Consequently we must have $\xi^{0} X^{\prime \mu}+\xi^{1} \Pi^{\mu} \in H^{1}\left(S^{1}\right)$ which is equivalent to

$$
\xi^{+}\left(\sigma^{+}\right)\left\{X^{\prime}(\tau, \sigma)+\Pi(\tau, \sigma)\right\}-\xi^{-}\left(\sigma^{-}\right)\left\{X^{\prime}(\tau, \sigma)-\Pi(\tau, \sigma)\right\} \in H^{1}\left(S^{1}\right)^{4}
$$

and this condition defines a vector space $V_{\tau} \subset H^{2}\left(S^{1}\right)^{4} \times H^{1}\left(S^{1}\right)^{4}$ for a fixed $\tau$. Hence the discontinuity of $\xi$ restricts the domain of $X_{Q_{\xi}}$, to the subspace ${ }^{* 7} V_{\tau}$. In consequence the Poisson bracket $\left\{Q_{\xi}, Q_{\eta}\right\}$ with two discontinuous fields $\xi, \eta$ is not defined on the whole of $H^{2}\left(S^{1}\right)^{4} \times H^{1}\left(S^{1}\right)^{4}$.

Our next task is to examine strings with varying topology. We want $Q_{\xi}$ to be defined on a phase space $\mathcal{P}$ and from (14) it follows that $\mathcal{P}$ must depend on $C_{\tau}$ or at least on $C_{\tau_{c}}:=C_{\tau_{c}} \backslash\left\{p_{c}\right\}$, for critical values of $\tau$. Therefore we need a Hamiltonian description for each fixed manifold $(M, d k)$. The natural generalization of $P=H^{2}\left(S^{1}\right)^{4} \times L^{2}\left(S^{1}\right)^{4}$. is to take the collection of spaces $P_{\tau}=H^{1}\left(C_{\tau}\right)^{4} \times L^{2}\left(C_{\tau}\right)^{4}$ (the integration measure is defined by the conformal parameter $\sigma$ ). In the case of critical curves $C_{\tau_{c}}$ we compactify the different components of $C_{\tau_{c}}$ in such a way that the resulting space is isomorphic to $C_{\tau_{c}+\epsilon}$, where $\epsilon$ has to be sufficiently small. In fig.2, for example, one gets two copies of $S^{1}$ for the compactification of $C_{\tau_{c}}$ which we also denote by $C_{\tau_{c}}$. Now all $C_{\tau}$ are smooth compact manifolds (not submanifolds of $M$ for $\tau_{c}$ ) so one avoids the discussion of boundary values at $p_{c}$ and instead gets the notion of differentiability along $C_{\tau_{c}}$ which is necessary to

*7 One can identify $H^{1}\left(S^{1}\right)$ with a subspace of $C^{0}\left(S^{1}\right)$ due to the Sobolev lemma. If $\left.\xi\right|_{C_{\tau}}=\left.\xi^{+}\right|_{C_{\tau}}$ has only one discontinuity at $\sigma_{0}^{+}$the condition $(18)$ gives $X^{\prime \mu}\left(\tau, \sigma_{0}^{+}-\tau\right)+\Pi^{\mu}\left(\tau, \sigma_{0}^{+}-\tau\right)=0$, i.e. $V_{\tau} \neq H^{2}\left(S^{1}\right)^{4} \times H^{1}\left(S^{1}\right)^{4}$. 
understand what $H^{1}\left(C_{\tau_{c}}\right)$ means. A symplectic form $\omega_{\tau}$ on $P_{\tau}$ is obtained by formula (15) where now $<,>_{L^{2}}$ denotes the scalar product on $L^{2}\left(C_{\tau}\right)$. The Poisson bracket is defined by

$$
\{f, g\}_{\tau}=\omega_{\tau}\left(X_{f}, X_{g}\right) \quad, \quad X_{f}, X_{g} \in T P_{\tau} .
$$

Definition: $W e$ call $\mathcal{P}=\bigcup_{\tau \in \mathbb{R}}\left(P_{\tau}, \omega_{\tau}\right)$ the generalized phase space.

String branchings occur at times $\tau=\tau_{1}, . ., \tau_{k}$ and the spaces $C_{\tau}$ are naturally isomorphic if $\tau$ is restricted to $J_{0}=\left(-\infty, \tau_{1}\right), J_{i}=\left[\tau_{i}, \tau_{i+1}\right), i=1 . . k-1$ or $J_{k}=\left[\tau_{k}, \infty\right)$ : One simply identifies points with the same value of the conformal $\sigma$-parameter by maps $I_{\tau \tau^{\prime}}^{i}$ : $C_{\tau} \rightarrow C_{\tau^{\prime}}, \forall \tau, \tau^{\prime} \in J_{i}$. This yields isomorphisms $I_{\tau \tau^{\prime}}^{i *}: P_{\tau^{\prime}} \rightarrow P_{\tau}$ and they define an equivalence relation on $\bigcup_{\tau \in J_{i}} P_{\tau}$. Of course an analogous equivalence relation holds on the tangent spaces $\bigcup_{\tau \in J_{i}} T P_{\tau}$. We denote the space of equivalence classes by $P_{i}$ resp. $T P_{i}$ and a symplectic form $\omega_{i}$ on the class $P_{i}$ is defined by $\omega_{i}\left(X_{i}, Y_{i}\right):=\omega_{\tau}\left(X_{\tau}, Y_{\tau}\right)$ where $X_{\tau}, Y_{\tau} \in T P_{\tau}$ are the representing fields of $X_{i}, Y_{i} \in T P_{i}$ with arbitrary $\tau \in J_{i}$. The vectors $\left(\Pi, X^{\prime \prime}\right)_{(X, \Pi)} \in T_{(X, \Pi)} P_{\tau}$ are equivalent, hence there is a vector field $\left(\Pi, X^{\prime \prime}\right)_{i}$ on each class $P_{i}$ and the Hamiltonian flow $\Phi_{\tau}^{i}: P_{i} \rightarrow P_{i}$ yields integral curves on this space. The representing functions $\left(X_{\tau}, \Pi_{\tau}\right) \in P_{\tau}$ of such an integral curve define a section $s_{i}: J_{i} \rightarrow \bigcup_{\tau \in J_{i}} P_{\tau}$ which describes a string motion within the time interval $J_{i}$. At the critical value $\tau_{i+1}$ the $\operatorname{limit} \lim _{\tau \uparrow \tau_{i+1}} s(\tau)$ has to be interpreted as a function on $C_{\tau_{i+1}}$ and if this new initial condition is in $P_{\tau_{i+1}}$ dynamics continues via $\Phi_{\tau}^{i+1}$. Hence a string motion on $(M, d k)$ is given by a section ${ }^{* 8} s: \mathbb{R} \rightarrow \bigcup_{\tau \in \mathbb{R}} P_{\tau}$ and in order to be well-defined the transition conditions $\lim _{\tau \uparrow r_{i}} s(\tau) \in P_{\tau_{i}}$ must be satisfied. This clearly restricts the set of initial states $\left(X_{\tau_{0}}, \Pi_{\tau_{0}}\right)$ at $\tau_{0}<\tau_{1}$ for each fixed base manifold $(M, d k)$.

However these restrictions are not of physical nature: If the transition conditions fail for a given initial state it means that our choice $(M, d k)$ is not appropriate for the resulting string motion. The choice $M=\mathbb{R} \times S^{1}$ fits well for any initial state but from chapter 2 follows that there are also different possible choices in general. It is at this point that we have to consider the totality (or at least a subset) of compact Riemann surfaces, i.e. moduli space, in order to get a well-defined Hamiltonian description for arbitrary string motions.

We carried out this formal construction to adhere as close as possible at the usual Hamiltonian treatment which deals with integral curves in a fixed phase space. Notice that for $M=\mathbb{R} \times S^{1}$ we have $J_{0}=\mathbb{R}, P_{0} \simeq H^{1}\left(S^{1}\right)^{4} \times L^{2}\left(S^{1}\right)^{4}$ and $\omega_{0}$ is given by (15), hence our description reduces to the usual one for $M=\mathbb{R} \times S^{1}$. Now we study the algebra of conserved charges:

Theorem 4.3 The conformal algebra $\mathcal{A}_{M}$ has an anti-representation on each space $H^{2}\left(C_{\tau}\right)^{4} \times H^{1}\left(C_{\tau}\right)^{4}$ via Poisson brackets of the associated charges $Q_{\xi}$ :

$$
\left\{Q_{\xi}, Q_{\eta}\right\}_{\tau}=-Q_{[\xi, \eta]} \quad \forall \xi, \eta \in \mathcal{A}_{M}
$$

\footnotetext{
*8 By "section" we only mean $g(\tau) \in P_{\tau}$ : The set $\cup_{\tau \in \mathbb{R}} P_{\tau}$ hás no canonical smooth vector bundle structure because the spaces $P_{\tau}$ and $P_{\tau^{\prime}}$ are not naturally isomorphic for arbitrary $\tau, \tau^{\prime}$.
} 
Proof: Formula (19) and the generalization of Lemma 4.1 ( $S^{1}$ is substituted by $C_{\tau}$ ) yield:

$$
\left\{Q_{\xi}, Q_{\eta}\right\}_{\tau}=<Y_{\xi}, Z_{\eta}>_{L^{2}}-<Z_{\xi}, Y_{\eta}>_{L^{2}}=\int_{C_{\tau}}\left(\xi^{0} \Pi+\xi^{1} X^{\prime}\right)\left(\eta^{0} X^{\prime}+\eta^{1} \Pi\right)^{\prime} d \sigma-(\xi \leftrightarrow \eta)
$$

where $\xi \leftrightarrow \eta$ denotes the same integral expression with $\xi$ and $\eta$ interchanged. After some elementary calculations we find for $\left\{Q_{\xi}, Q_{\eta}\right\}_{\tau}$ :

$$
\int_{C_{\tau}}\left\{\left(\xi^{0} \partial_{1} \eta^{0}+\xi^{1} \partial_{1} \eta^{1}\right) \Pi X^{\prime}-\frac{1}{2}\left(\eta^{0} \partial_{1} \xi^{1}-\xi^{1} \partial_{1} \eta^{0}\right) X^{\prime 2}+\frac{1}{2}\left(\xi^{0} \partial_{1} \eta^{1}-\eta^{1} \partial_{1} \xi^{0}\right) \Pi^{2}\right\} d \sigma-(\xi \leftrightarrow \eta)
$$

We now use (13) to substitute the first term in each bracket e.g. $\xi^{0} \partial_{1} \eta^{0}=\xi^{0} \partial_{0} \eta^{1}$ and after collecting analogous expressions from $\xi \leftrightarrow \eta$ the assertion follows.

Remark 1: An important step in the proof is the substitution of terms like $\xi^{0} \partial_{1} \eta^{0}$ by $\xi^{0} \partial_{0} \eta^{1}$ because only in this way one gets the $\tau$-derivatives for the Lie bracket $[\xi, \eta]$. This is only possible if (13) is fulfilled, i.e. only if the functions $\xi^{0}$ and $\xi^{1}$ can be interpreted as the restriction of the components of a conformal vector field $\xi$ on $M$. For general fields $\xi$, $\eta$ on $M$ the substitution is not allowed but we can take a bit different point of view: At fixed $\tau_{0}$ we have $\left.\xi\right|_{\tau_{0}}(p)=\xi^{+}(p) \partial_{\sigma^{+}}+\xi^{-}(p) \partial_{\sigma^{-}}$and there is a unique conformal extension $\xi_{c}$ (constructed by parallel translations) of these initial data within some interval $\left[\tau_{0}, \tau_{0}+\epsilon\right.$ ):

$$
\xi_{c}(\tau, \sigma):=\xi^{+}\left(\tau_{0}, \sigma+\Delta \tau\right) \partial_{\sigma^{+}}+\xi^{-}\left(\tau_{0}, \sigma-\Delta \tau\right) \partial_{\sigma^{-}} \quad, \quad \tau=\tau_{0}+\Delta \tau \in\left[\tau_{0}, \tau_{0}+\epsilon\right)
$$

We may not substitute $\xi^{0} \partial_{1} \eta^{0}$ by $\xi^{0} \partial_{0} \eta^{1}$ but instead we can take $\xi_{c}^{0} \partial_{0} \eta_{c}^{1}$ and this yields

Corollary 4.4: Let $\xi$ and $\eta$ be arbitrary smooth vector fields on $M$. Then the Poisson bracket $\left\{Q_{\xi}, Q_{\eta}\right\}_{\tau}(16)$ is given on $H^{2}\left(C_{\tau}\right)^{4} \times H^{1}\left(C_{\tau}\right)^{4}$ by

$$
\left\{Q_{\xi}, Q_{\eta}\right\}_{\tau}=-Q_{\left[\xi_{c}, \eta_{c}\right]}
$$

Here $\xi_{c}$ and $\eta_{c}$ denote the conformal extension (22) arising from $C_{\tau}$.

Notice that 4.4 remains valid for complex valued vector fields, i.e. $\xi_{p}, \eta_{p} \in T_{p} M \otimes \mathbb{C}$.

Remark 2: To obtain (21) we had to integrate by parts:

$$
\int_{C_{\tau}}\left\{\xi^{1} \eta^{0}\left(X^{\prime 2}\right)^{\prime}+\xi^{0} \eta^{1}\left(\Pi^{2}\right)^{\prime}\right\} d \sigma=-\int_{C_{\tau}}\left\{\left(\xi^{1} \eta^{0}\right)^{\prime} X^{\prime 2}+\left(\xi^{0} \eta^{1}\right)^{\prime} \Pi^{2}\right\} d \sigma
$$

If $\xi^{1} \eta^{0}$ or $\xi^{0} \eta^{1}$ were discontinuous along $\gamma_{c}^{+}$or $\gamma_{c}^{-}$we would obtain additional boundary values, e.g. at $\tau=0$ terms like $\Pi^{2}(0,0) \cdot\left(\xi^{0} \eta^{1}\left(0, \sigma_{0}\right)-\xi^{0} \eta^{1}(0,0)\right)$. For critical values $\tau_{c}$ one can give explicit examples where these additional terms do not cancel at certain points $(X, \Pi) \in V_{\tau_{c}}(18)$. This means that we have

$$
\left\{Q_{\xi}, Q_{\eta}\right\}_{\tau_{c}}=-Q_{[\xi, \eta]}+\text { extra term }
$$

at these points; notice that the derivatives occuring in $[\xi, \eta]$ are interpreted as left-sided resp. right-sided limits at those points $(\tau, \sigma)$ where $\left.\xi\right|_{C_{\tau}}$ or $\left.\eta\right|_{\tau}$ are discontinuous. Equivalently we may drop the extra term and interpret the Lie bracket $[\xi, \eta]$ in a distributional 
sense. In consequence the simple algebraic structure of Virasoro generators $e^{i n \sigma^{+}} \partial_{\sigma^{+}}$is lost on the level of Poisson brackets if we work not on $\mathbb{R} \times S^{1}$ but e.g. on the manifold pictured in fig.2. This is the main reason why one looks for smooth basis fields $\xi^{ \pm}$. An elegant solution for this problem is provided by $\mathrm{KN}$-theory, using the holomorphic structure of $M$.

Our final observation about the proof of 4.3 is that we made no explicit use of the fact $\xi^{ \pm}\left(\gamma_{c}^{ \pm}\right)=0$. Only continuity of $\xi$ and $\eta$ is necessary for the vanishing of boundary terms:

Corollary 4.5 The algebra $\mathcal{A}_{M}$ of smooth vector fields on $\stackrel{M}{M}$ which obey (13) is represented on each space $H^{2}\left(C_{\tau}\right)^{4} \times H^{1}\left(C_{\tau}\right)^{4}$ via Poisson brackets by formula (20).

The vector fields in $\mathcal{A}_{M}$ are generally not smooth fields on $M$ but each $\xi \in \mathcal{A}_{M}$ generates a local conformal flow on $\dot{M}$ and this suffices to obtain a conserved quantity $Q_{\xi}$. Therefore it is $\mathcal{A}_{M}$ which is of physical interest ${ }^{* 9}$.

\section{The classical background of $\mathrm{KN}$-theory}

In this chapter we restrict the discussion to the case of one incoming and one outgoing string because we will need two analytical theorems that are available in this case (expansions (24) and (25) below, for generalizations see [9]). We denote the space of $C^{\infty}$-smooth complex valued vector fields on $C_{\tau}$ by $\mathcal{L}\left(C_{\tau}\right)$ and the space of meromorphic vector fields on $\bar{M}$ that are holomorphic on $M$ by $\mathcal{L}(M)$. Around each point $p \neq p_{c}$ there are holomorphic $(\tau, \sigma)$ coordinates defined via the differential $d k$ and any vector field $e \in \mathcal{L}(M)$ is represented by $l(\tau+i \sigma) \frac{1}{2}\left(\partial_{\tau}-i \partial_{\sigma}\right)$. If we fix $\tau \neq \tau_{c}$ and take $\sigma$ as coordinate along $C_{\tau}$ we have a natural "restriction"

$$
r_{\tau}:\left\{\begin{array}{ccc}
\mathcal{L}(M) & \rightarrow & \mathcal{L}\left(C_{\tau}\right) \\
l(\tau+i \sigma) \frac{1}{2}\left(\partial_{\tau}-i \partial_{\sigma}\right) & \rightarrow & -i l(\tau+i \sigma) \partial_{\sigma}
\end{array}\right.
$$

which is a Lie algebra homomorphism. A fundamental theorem about the set $r_{\tau}(\mathcal{L}(M))$ states the following [7]: There exists a countable basis $\left\{e_{n}\right\}$ of $\mathcal{L}(M)$ such that each field $v_{\tau} \in \mathcal{L}\left(C_{\tau}\right)$ can be expanded in a uniformly convergent series:

$$
v_{\tau}=\sum_{-\infty}^{\infty} a_{n} \cdot r_{\tau}\left(e_{n}\right)
$$

Remark: The map $r_{\tau}$ can also be defined for critical values $\tau_{c}$ but then the expansion (24) (in the sense of uniform convergence) is not generally valid: An arbitrary $e \in \mathcal{L}(M)$ reads $e=l(z) \partial_{z}$ in holomorphic $z$-coordinates around $p_{c}$. For $p \neq p_{c}$ we choose $u$-coordinates $u=\tau+i \sigma=\int_{z_{0}}^{z} d k\left(z^{\prime}\right) d z^{\prime}$ so we have $e=l(u) \partial_{u}=l(z) \partial_{z}$ with $l(u)=l(z) d k(z)$. Since $d k$ vanishes at $p_{c}$ the restriction $r_{\tau}(e)(p)$ converges to zero for $p \rightarrow p_{c}$ and consequently at most those fields $v_{\tau_{c}}$ on $C_{\tau_{c}}$ can be expanded that obey $v_{\tau_{c}}\left(p_{c}\right)=0$.

\footnotetext{
*9 The situation is similar in an Euclidean context: A holomorphic vector field on $\mathbb{P}^{1} \backslash\{0, \infty\}$ is a conformal-field with respect to the Euclidean metric $d z d \bar{z}$ (on $\mathscr{C}$ ) but only those fields which are holomorphic on $\mathbb{P}^{1}$ generate global conformal transformations.
} 
A representation analogous to (24) holds with meromorphic quadratic differentials $\Omega$ on $\bar{M}$ (i.e. locally $\Omega=f(z) d z^{2}$ ) that are holomorphic on $M$ : Let $\left\{\Omega^{m}\right\}$ be the dual basis to $\left\{e_{n}\right\}$ with respect to the pairing $\langle\Omega, e\rangle:=\frac{1}{i} \int_{C_{\tau}} \Omega(e, \cdot)$, i.e. we have $\left.<\Omega^{m}, e_{n}\right\rangle=\delta_{n}^{m}$; $\left\{\Omega^{n}\right\}$ was introduced in [8], up to a factor $2 \pi$. Then for any smooth quadratic differential $\Omega_{\tau}$ on $C_{\tau}$ there is an expansion $\left(i_{\tau}: C_{\tau} \hookrightarrow M\right.$ denotes the embedding)

$$
\Omega_{\tau}=\sum_{-\infty}^{\infty} b_{n} \cdot i_{\tau}^{*} \Omega^{n}
$$

With these analytical preliminaries we now describe the physically relevant quantities in a convenient way: In local light cone coordinates $\sigma^{ \pm}=\tau \pm \sigma$ the energy momentum tensor is of the form

$$
T=T_{++} d \sigma^{+} \otimes d \sigma^{+}+T_{--} d \sigma^{-} \otimes d \sigma^{-}
$$

with $T_{++}=\partial_{+} X \partial_{+} X$ and $T_{--}=\partial_{-} X \partial_{-} X$. For an arbitrary conformal vector field $\xi=\xi^{+} \partial_{\sigma^{+}}+\xi^{-} \partial_{\sigma-}$ the quantity $Q_{\xi}$ is given by

$$
Q_{\xi}=Q_{\xi^{+}}+Q_{\xi^{-}}=-\int_{C_{\tau}} \xi^{+} T_{++} d \sigma-\int_{C_{\tau}} \xi^{-} T_{--} d \sigma
$$

Due to (24) the field $\xi_{\tau}^{+}(\sigma):=\xi^{+}(\tau+\sigma) \partial_{\sigma}$ along $C_{\tau}$ has an expansion $\xi_{\tau}^{+}=\sum a_{n} r_{\tau}\left(e_{n}\right)=$ $\sum a_{n} \cdot(-i) l_{n}(\tau+i \sigma) \partial_{\sigma}$ which applies for any value $\tau \neq \tau_{c}$, and we receive:

$$
Q_{\xi^{+}}=\frac{1}{i} \sum_{-\infty}^{\infty} a_{n}(\tau) \int_{C_{\tau}}-l_{n}(\tau+i \sigma) T_{++} d \sigma=: \frac{1}{i} \sum_{-\infty}^{\infty} a_{n}(\tau) L_{e_{n}}
$$

Therefore we can evaluate $\left\{Q_{\xi^{+}}, Q_{\eta^{+}}\right\}_{\tau}$ if we know the Poisson brackets $\left\{L_{e_{n}}, L_{e_{m}}\right\}_{\tau}$.

Proposition 5.1 The functions $L_{e_{n}}=-\int_{C_{\tau}} l_{n}(\tau+i \sigma) T_{++} d \sigma$, defined on $P_{\tau}$, obey:

$$
\left\{L_{e_{n}}, L_{e_{m}}\right\}_{\tau}=-i L_{\left[e_{n}, e_{m}\right]} \text {. }
$$

Proof: In view of Corollary 4.4 we have to consider the vectors $e_{n}^{+}:=l_{n}\left(\tau_{0}+i \sigma\right) \partial_{\sigma^{+}}$, that are defined on $C_{\tau_{0}}$. Their local conformal extension (22) is given by

$$
e_{n_{c}}^{+}(\tau, \sigma)=l_{n}\left(\tau_{0}+i(\sigma+\Delta \tau)\right) \partial_{\sigma^{+}} \quad, \quad \tau=\tau_{0}+\Delta \tau .
$$

At $\tau=\tau_{0}$ we have $L_{e_{n}}=Q_{e_{n_{c}}^{+}}$and the derivative $\partial_{\sigma^{+}}$acts like $i \partial_{z}=\frac{i}{2}\left(\partial_{\tau}-i \partial_{\sigma}\right)$ on $l$,

$$
\left.\partial_{\sigma^{+}} l\left(\tau_{0}+i(\sigma+\Delta \tau)\right)\right|_{\tau=\tau_{0}}=i \frac{\partial l}{\partial z}\left(\tau_{0}+i \sigma\right) .
$$

This implies $\left[e_{n_{c}}^{+}, e_{m_{c}}^{+}\right]=i\left[e_{n}, e_{m}\right]_{c}^{+}$for $\tau=\tau_{0}$. Using Corollary 4.4 we obtain

$$
\left\{L_{e_{n}}, L_{e_{m}}\right\}_{\tau_{0}}=\left\{Q_{e_{n}^{+}}, Q_{e_{m}^{+}}\right\}_{\tau_{0}}=-Q_{\left[e_{n_{c}}^{+}, e_{m_{c}}^{+}\right]}=-Q_{i\left[e_{n}, e_{m}\right]_{c}^{+}}=-i L_{\left[e_{n}, e_{m}\right]} .
$$

Meromorphic vector fields $e_{n}$ obey the KN algebra $\left[e_{n}, e_{m}\right]=\sum_{j} C_{n m}^{j} e_{n+m+j}$ where $C_{n m}^{j}$ are structure constants and the summation ranges over some fixed, finite set [7]. Hence (27) reads $\left\{L_{e_{n}}, L_{e_{m}}\right\}_{\tau}=-i \sum_{j} C_{n m}^{j} L_{e_{n+m+j}}$ and we find that the $L_{e_{n}}$ satisfy a Poisson algebra 
which is not $\tau$-dependent. This is somewhat astonishing because meromorphic vector fields do not correspond to a symmetry of $\widetilde{S}(12)$. We interpret this representation property as follows: The conformal extension (28) is constructed via the map

$$
W_{\tau_{0}}:\left(\tau_{0}+\Delta \tau+i \sigma\right) \rightarrow\left(\tau_{0}+i(\sigma+\Delta \tau)\right)
$$

defined on some right-sided neighbourhood $U_{\tau_{0}}=\bigcup_{\tau \in\left[\tau_{0}, \tau_{0}+\epsilon\right)} C_{\tau}$ of $C_{\tau_{0}} . W_{\tau_{0}}$ may be regarded as the local analogy of a Wick rotation: In essence we substitute $\Delta \tau$ by $i \Delta \tau$. The map $W_{\tau_{0}}$ together with the substitution $\frac{1}{2}\left(\partial_{\tau}-i \partial_{\sigma}\right) \rightarrow-i \partial_{\sigma}+$ results in a homomorphism from $\mathcal{L}(M)$ to the space of complex valued conformal vector fields on $U_{\tau_{0}}$. We made use of this homomorphism (up to a factor $i$ ) in the proof of 5.1. Notice that the concept of a Wick rotation only makes sense because there is a canonical splitting of the Riemann surface into time $\times$ space, induced by the differential $d k$.

Remark: The functions $L_{e_{n}}$ and $Q_{\xi}(\xi \in \mathcal{A})$ vanish on-shell because the constraints $T=0$ are conserved in time. Even without these constraints $Q_{\xi}$ would be a conserved quantity (due to Noether's theorem). This property does not hold for $L_{e_{n}}$ : For $T \neq 0$ these functions depend on time $\tau$.

Finally we interpret the $L_{e_{n}}$ by means of the pullback $i_{\tau}^{*} T$ of the energy momentum tensor along $C_{\tau}$. The local coefficient of $i_{\tau}^{*} T$ is given by $i_{\tau}^{*} T\left(\partial_{\sigma}, \partial_{\sigma}\right)=T_{++}(\tau, \sigma)+T_{--}(\tau, \sigma)$ hence the tensor splits into two parts:

$$
i_{\tau}^{*} T=T_{++} d \sigma^{2}+T_{--} d \sigma^{2}=: T_{+}+T_{-} .
$$

Proposition 5.2 The $T_{+}$part of $i_{\tau}^{*} T$ can be decomposed into $T_{+}=\sum_{-\infty}^{\infty} L_{e_{n}} \cdot i_{\tau}^{*} \Omega^{n}$ :

Proof: $T_{+}$is a quadratic differential on $C_{\tau}$ so there is an expansion (25) $T_{+}=\sum b_{m} i_{\tau}^{*} \Omega^{m}$. We can express $L_{e_{n}}$ via $T_{+}, L_{e_{n}}=\frac{1}{i} \int_{C_{\tau}} T_{+}\left(r_{\tau}\left(e_{n}\right), \cdot\right)$, which gives:

$$
\begin{aligned}
L_{e_{n}} & =\frac{1}{i} \int_{C_{\tau}} \sum b_{m} i_{\tau}^{*} \Omega^{m}\left(r_{\tau}\left(e_{n}\right), \cdot\right)=\sum b_{m} \frac{1}{i} \int_{C_{\tau}} \Omega^{m}(\tau+i \sigma) l_{n}(\tau+i \sigma) i d \sigma \\
& =\sum b_{m} \frac{1}{i} \int_{C_{r}} \Omega^{m}\left(e_{n}, \cdot\right)=\sum b_{m} \delta_{n}^{m}=b_{n} .
\end{aligned}
$$

Hence the $T_{+}$-components $L_{e_{n}}$ of the energy momentum tensor represent the algebra $\mathcal{L}(M)$ on each space $\left(P_{\tau}, \omega_{\tau}\right)$, for $\tau \neq \tau_{c}$.

Example: Let us distinguish explicitly between the usual theory on $\mathbb{R} \times S^{1}$ and the treatment given here. Usually one uses the basis

$$
\xi_{n}^{+}:=e^{i n(\tau+\sigma)} \partial_{\sigma^{+}}, n \in \mathbb{Z}
$$

for global $\xi^{+}$-fields on $\mathbb{R} \times S^{1}$, satisfying (13). Each conformal vector field $\xi^{+}$admits a Fourier decomposition $\xi^{+}=\sum_{-\infty}^{\infty} a_{n} \xi_{n}^{+}$with time independent coefficients $a_{n}$. The conserved charges read $Q_{\xi_{n}^{+}}=-\int e^{i n(\tau+\sigma)} T_{--}(\tau, \sigma) d \sigma$. 
In our treatment we start with $\bar{M}=\mathbb{P}^{1}$ and we choose $P_{1}=0$ and $Q_{1}=\infty$. A basis of holomorphic vector fields on $M=\mathbb{P}^{1} \backslash\{0, \infty\}$ is given by

$$
e_{n}:=z^{n+1} \partial_{z}, n \in \mathbb{Z} \text {. }
$$

The differential $d k=\frac{1}{z} d z$ defines the $(\tau, \sigma)$-representation of $e_{n}=e^{n(\tau+i \sigma)} \frac{1}{2}\left(\partial_{\tau}-i \partial_{\sigma}\right)$ and from (28) we obtain $\widehat{e}_{n_{c}}^{+}=e^{n\left(\tau_{0}+i(\sigma+\Delta \tau)\right)} \partial_{\sigma^{+}}$. At $\tau=\tau_{0}$ this gives

$$
L_{e_{n}}=-e^{n \tau_{0}} \cdot \int_{0}^{2 \pi} e^{i n \sigma} T_{++}\left(\tau_{0}, \sigma\right) d \sigma=e^{n \tau_{0}(1-i)} Q_{\xi_{n}^{+}} .
$$

The time dependence drops out in the Poisson algebra $\left\{L_{e_{n}}, L_{e_{m}}\right\}_{\tau_{0}}=i(n-m) L_{e_{n+m}}$ because both sides contain the factor $e^{(n+m) \tau_{0}(1-i)}$. It is this observation which is generalized to arbitrary compact Riemann surfaces.

The whole reasoning for $\xi^{+}, T_{+}$can be repeated for $\xi^{-}, T_{-}$by simply using the antiholomorphic bases $\left\{\bar{e}_{n}\right\}$ and $\left\{\bar{\Omega}^{m}\right\}$. The resulting components of $T_{-}=\sum_{-\infty}^{\infty} L_{\bar{e}_{n}} \bar{\Omega}^{n}$ obey

$$
\left\{L_{\bar{e}_{n}}, L_{\bar{e}_{m}}\right\}_{\tau}=-i \sum_{j} \bar{C}_{n m}^{j} L_{\bar{e}_{n+m+j}}
$$

where $C_{n m}^{j}$ denote the $\mathrm{KN}$ structure constants of $\left[e_{n}, e_{m}\right]$. We also obtain

$$
\left\{L_{e_{n}}, L_{\bar{e}_{m}}\right\}_{\tau}=0 \text {. }
$$

There are two major advantages in dealing with $L_{e_{n}}$ and $L_{\bar{e}_{m}}$ instead of $Q_{\xi^{\ddagger}}$ :

1. The algebraic structure of $\left\{L_{e_{n}}\right\}$ is related (by complex conjugation) to the structure of $\left\{L_{\bar{e}_{m}}\right\}$. Such a property does not hold for conformal vector fields $\xi^{ \pm}$: Generally there is no canonical map which assigns $\xi^{+}$-fields to $\xi^{-}$-fields because the conditions $\xi^{ \pm}\left(\gamma_{c}^{ \pm}\right)=$ const. are totally different.

2. For any Riemann surface $\bar{M}$ the algebra $\mathcal{L}(M)$ is generalized graded and different representations (for central extensions of $\mathcal{L}(M)$ ) are available in that case $[7,10]$. An analogous grading for the algebra $\mathcal{A}_{M}$ is not obvious.

With these considerations we conclude our treatment of the classical theory. It follows from the Poisson structure and the Hamiltonian formulation that this description is directly. related to $\mathrm{KN}$-theory.

\section{Concluding remarks}

The description of string dynamics given here improves the physical understanding in the following sense: First, it showes how branching processes emerge naturally from a geometric point of view. Second, it makes transparent the relation between the conformal symmetry of a topological non-trivial string world sheet and the holomorphic structures on the associated Riemann surface. This treatment differs from that one where the "Euclidean version" of string theory is considered (cf. [12]): In the latter 'case one has a conformal symmetry with respect to an Euclidean metric $\delta$ on $M$ that locally reads $\delta(z)=e^{|\Phi(z)|} d z d \bar{z}$. 
The corresponding conserved charges $\widetilde{L}_{e_{n}}$ represent (as expected) the Euclidean conformal symmetry on phase space.

In our description not only the conformal symmetry of $(M, \eta)$ is realized by the $Q_{\xi}$ : Also the symmetry of $(M, \delta)$ is represented via "charges" $L_{e_{n}}$; the connection between these two structures is exploited by the theory of Krichever and Novikov.

\section{Acknowledgement}

I thank Ernst Binz for helpful discussions. Special thanks are due to Günter Schwarz for encouraging me to set up a rigorous Hamiltonian description. This work was supported in part by the GradFöG Fund of Baden-Württemberg.

\section{References}

1. J.Scherk, An Introduction to the Theory of Dual Models and Strings, Rev. Mod. Phys. 47, (1975) 123-164.

2. L. Brink and M. Henneaux, Principles of String Theory, Plenum Press, New York, (1988).

3. N. Turok, Grand Unified Strings and Galaxy Formation, Nucl. Phys. B242, (1984) 520541.

4. D. Garfinkle and T.Vachaspati, Radiation from Kinky, Cuspless Cosmic Loops, Phys. Rev. D 36, (1987) 2229-2241.

5. T. Kornhaß, Klassische Erhaltungsgrößen auf verzweigten Stringtrajektorien der Nambu-Goto Theorie, Dissertation, Freiburg (1991), THEP 91/9.

6. S.B. Giddings and S.A. Wolpert, A Triangulation of Moduli Space from Light-Cone String Theory, Com. Math. Phys. 109, (1987) 177-190.

7. I.M Krichever and S.P. Novikov, Algebras of Virasoro Type, Riemann Surfaces and Structures of the Theory of Solitons, Funk. Anal. i Prilozhen 21 (2), (1987) 46-63.

8. I.M. Krichever and S.P. Novikov, Virasoro Type Algebras, Riemann Surfaces and Strings in Minkowski Space, Funktsional. Anal. i Prilozhen 21 (4), (1987) 47-61.

9. V.A. Sadov, Bases of Multipunctured Riemanin Surfaces and Interacting String Amplitudes, Com. Math. Phys. 136, (1991) 585-597.

10. M. Schlichenmaier, Central Extensions and Semi-infinite Wedge Representations of Krichever-Novikov Algebras for. More Than two Points, Lett. Math. Phys. 20, (1990) 33-46.

11. A. Ruffing, T. Deck and M. Schlichenmaier, String Branchings on Complex Tori and Algebraic Representations of Generalized Krichever-Novikov Algebras, Lett. Math. Phys. 26, (1992) 23-32.

12. A. Lugo and J. Russo, Hamiltonian Formulation of Scattering Amplitudes in String Theory at Genus g, Nucl. Phys. B 322, (1989) 210-234.

13. R. Dick, Topological Aspects of Chiral Fields in Two Dimensions and Superstring Vertices, Universität München, LMU-TPW 91-10, (1991).

14. R. Dick, Conformal Gauge Fixing in Minkowski Space, Lett. Math. Phys. 18, (1989) 67-76.

15. K. Meada and N. Turok, Finite Width Corrections to the Nambu Action for the NielsenOlesen String, Phys. Let. B 202, (1988) 376-380.

16. P. Chernoff and J. Marsden, Properties of infinite dimensional Hamiltonian systems, Lec. Notes Math. 425 (1974). 